\section{Clinical Emergency Radiology}

J.C. Fox, Ed.

Cambridge, U.K.: Cambridge University Press, 2017, 643 pages, $\$ 165$

Technologic advances in imaging and the use of ultrasonography, MRI, and CT have revolutionized the way acute injuries and conditions are managed in the emergency room. Emergency radiology, a subspecialty of radiology that deals with the diagnosis of the acutely ill or traumatized patient in the emergency department, plays a vital role in the timely evaluation of emergency patients from head to toe. Plain radiography remains the imaging study of choice for most trauma of the extremities and to search for a foreign body in a wound. The chest radiograph is also the most commonly ordered test in the emergency department to evaluate chest pain, breathing complaints, trauma, fever, and altered mental status. Although abdominal imaging is now largely performed using CT, MRI, or ultrasonography, plain film radiography can still be valuable in providing specific answers to urgent clinical questions related to acute abdominal pain, vomiting, trauma or fever. Nothing has generated as much change in emergency medicine in the past 20 years as the introduction of bedside portable ultrasonography systems, because they can answer clinical questions immediately, accelerating both decision making and treatment. Ultrasonography also benefits many procedures commonly performed in the emergency department. Emergency physicians routinely obtain CT scans to evaluate many types of patients in daily practice, such as those who present with abdominal or chest pain and neurologic symptoms. CT of the head is one of the most common imaging studies ordered in the emergency department. CT of the spine is also becoming common, as it is more sensitive than plain radiography for many conditions, is less expensive and faster than MRI, and usually is available at all hours of the day. Since its inception, CT has greatly aided in rapid diagnosis of abdominal and pelvic disease. MRI is quickly becoming the preferred alternative for evaluating certain complaints, although CT continues to be the diagnostic imaging modality of choice for many clinical situations.

This second edition of Clinical Emergency Radiology, written by the editor and 73 contributors, is composed of 4 parts with 43 chapters, just like the first edition in 2008. Part 1 (11 chapters) deals with plain radiography of the upper and lower extremities, chest, abdomen, and spine. There are special chapters on extremity studies in the elderly and in abused pediatric patients. Part 2 (16 chapters) discusses the physics of ultrasonography, followed by biliary, cardiac, genitourinary, gastrointestinal, aortic, ocular, and musculoskeletal ultrasonography and the utility of ultrasonography in trauma, deep vein thrombosis, and resuscitation. Part 3 (10 chapters) demonstrates CT of the spine, head, face, chest, abdomen, and pelvis, as well as CT angiography of the chest, abdomen, head and neck, and extremities. The risks of intravenous contrast material in patients susceptible to radiographic contrast-induced nephropathy are discussed in chapter 28. Part 4 (6 chapters) handles the physics of MRI followed by MRI of the brain, spine, chest, heart, abdomen, and extremities. The full spectrum of conditions diagnosed by each modality is covered in detail, and examples of normal radiologic anatomy, patterns, and anomalies are also included. Covering topics ranging from ultrasonography at the point of clinical care to the interpretation of CT and MRI results, this book contains more than 2,200 clear images, each with detailed captions and line art that highlights key findings. Within each section, particular attention is devoted to practical tricks of the trade and tips for avoiding common pitfalls. The references are updated, and the index is useful.

This book is a new clinical resource in the field of emergency radiology and comprehensively covers both the technical application and the interpretation of all imaging studies used in the emergency room. It is a highly visual guide to the radiographic and advanced imaging modalities such as CT and MRI that are frequently used by physicians during the treatment of emergency patients. It is a useful resource for clinicians, residents, mid-level providers, and medical students who want to maximize the diagnostic accuracy of each modality for emergency patients without losing valuable time. I highly recommend this book to any medical professionals dealing with emergency patients, as well as to scientists studying emergency medicine.

\author{
E. Edmund Kim \\ University of California at Irvine \\ 101 The City Dr. S. \\ Orange, $C A 92868$ \\ E-mail: edmundek@uci.edu
}

